\title{
Estrategias de comunicación de los museos nacionales
} Communication Strategies of the National Museums

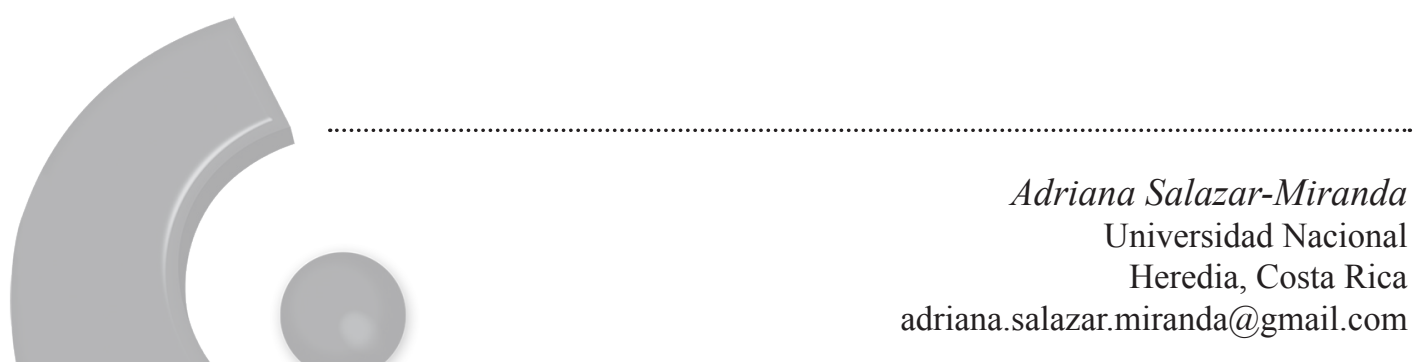

Recibido: 09/ 22/2014 Aceptado: 06/27/2016

Resumen. El objetivo es estudiar las estrategias de comunicación que emplea el Museo Nacional de Costa Rica, los Museos del Banco Central y el Museo de Arte Costarricense, ubicados en la zona central de la provincia de San José en Costa Rica, con el fin de conocer las estrategias que utilizan para brindar acceso y dar información a diversos grupos poblacionales sobre la oferta museística que ofrecen. El problema de estudio es que uno de los retos que enfrentan los museos es asegurar la visitación permanente y dinámica, a fin de preservar y asegurar su sostenibilidad y razón de ser, ante lo cual la comunicación se convierte en un puente entre los museos y los públicos. Los resultados más interesantes: los tres museos han implementado diversas acciones de comunicación, similares en estrategia; sin embargo, enfrentan una serie de retos en cuanto al monitoreo y la evaluación del impacto de lo realizado, escaso personal en los departamentos de comunicación, limitados recursos económicos y escaso conocimiento de los perfiles de los públicos visitantes.

Palabras claves: museos, estrategias de comunicación, sociología. 


\begin{abstract}
The objective is to study the communication strategies implemented by the National Museum of Costa Rica, the Central Bank Museums and the Museum of Costa Rican Art, located in the central area of San Jose province, Costa Rica, in order to know the strategies used to provide access and give information on their museum offer to several population groups.

The problem under study is that one of the challenges the museums face is to ensure permanent and dynamic visits in order to preserve and ensure their sustainability and raison d'être, to which the communication becomes a bridge between the museum and the public. The following are the most interesting results: the three museums have implemented several communication actions with similar strategies; however, they face a number of challenges in terms of monitoring and evaluating the impact of what has been done, the scarce staff in the departments of communication, limited economic resources, and little knowledge of the profiles of the public visitors.
\end{abstract}

Keywords: museums, communication strategies, sociology.

\title{
El objeto de estudio
}

El caso a presentar sintetiza lo realizado en el eje de estudio que versa sobre las estrategias de comunicación que se han trabajado desde el Proyecto Evaluación interdisciplinar de museos nacionales, ${ }^{I}$ de la Escuela de Sociología de la Universidad Nacional de Costa Rica. Y se enmarca dentro del objetivo específico que buscaba "determinar la correspondencia entre las estrategias de comunicación que emplean los museos y los grupos a los cuales impactan". Para ello fue necesario hacer, inicialmente, un estudio para determinar el perfil del público consumidor de dichos museos $\mathrm{y}$, en segundo lugar, hacer una indagación sobre las estrategias de comunicación que se están utilizando. Sobre esta segunda investigación, nace el presente artículo como un análisis inicial de la información obtenida, que tuvo como objetivo reflexionar sobre las estrategias de comunicación que emplean los museos en estudio, con el fin de ver cuáles utilizan actualmente para brindar acceso y dar información a diversos grupos poblacionales sobre la oferta museística que ofrecen.

\footnotetext{
Agradezco el apoyo de todo el equipo de investigación del Proyecto que de alguna u otra manera ayudaron con la investigación que alimenta el presente trabajo (Investigadores titulares: Adriana Salazar Miranda, Álvaro Madrigal Mora, Antonio Delgado Ballestero. Asistentes del proyecto de investigación: Mayra Brown Vargas, Ashley Charles Harvy, Laura Pérez Garbanzo y Mariam Rojas Quirós). En especial a Laura Pérez Garbanzo por su colaboración en toda la fase de recolección de información, así como a Ashley Charles Harvy por su colaboración en la búsqueda bibliográfica.
} 
El trabajo se realizó en tres museos: el Museo Nacional de Costa Rica, Museos del Banco Central de Costa Rica y Museo de Arte Costarricense. Los tres se han seleccionado por su posibilidad como futuros agentes dinamizadores, debido a que gozan de una importante trayectoria, una consolidación y sostenibilidad importante en el corto, mediano y largo plazo; la diversidad temática que trabajan, los públicos meta, cantidad de visitantes, arquitectura, formas de organización, tamaño y, además, una ubicación sociogeográfica relevante, dado que se ubican en el centro de la provincia principal o capital del país, denominada, San José.

Las preguntas de investigación fueron: ¿Definen los museos planes estratégicos de comunicación? ¿Cuáles son las áreas estratégicas de comunicación que trabajan? ¿Quiénes y en qué condiciones se ejecutan? ¿Cuáles son las estrategias de divulgación de información que utilizan dentro y fuera del museo? ¿Cuáles medios informativos utilizan? ¿Tienen los museos definidos cuáles son los públicos prioritarios a los que quieren llegar? ¿Cuáles son los medios más efectivos? ¿Cómo se mide el impacto de las estrategias ejecutadas?

\section{Marco del análisis}

Tal como lo indica el Consejo Internacional de Museos (ICOM), el concepto y la definición de museo ha evolucionado acomodándose al ritmo de los cambios y transformaciones que viven las sociedades actuales, a fin de asegurar su pertinencia, coherencia y contribución social, cultural y educativa. Actualmente, tomando en cuenta los estatutos del ICOM adoptados durante la $22^{\mathrm{a}}$ Conferencia General de Viena (Austria) en 2007, el museo se define de la siguiente manera:

Es una institución permanente, sin fines de lucro, al servicio de la sociedad y abierta al público, que adquiere, conserva, estudia, expone y difunde el patrimonio material e inmaterial de la humanidad con fines de estudio, educación y recreo. (ICOM, 2007, p. 3)

Esta definición propone trabajar desde los museos para captar y traer a sus espacios y actividades a diversos públicos, pero, sobre todo, aquel público general que históricamente ha quedado relegado o excluido de la oferta museística, si se compara, por ejemplo con la participación que han tenido los públicos especializados y los públicos cultos. Esto supone el reto de que el museo debería ofrecerse "más que como museo educativo, como un lugar lleno de sorpresas e interrogantes, que despierta en el visitante el deseo de aprender y comprender en completa libertad" (Arias, 1990, p. 180). 
Revista Universidad en Diálogo • Vol. 6, N. 2 2, Julio-diciembre 2016, pp. 161-174

ISSN 2215-2849 • EISSN: 2215-4752

DOI: http://dx.doi.org/10.15359/udre.6-2.9

Para lograr esto, Arias (1990, pp.181-187) menciona que los museos pueden implementar varias acciones:

- Las acciones de estrategias de captación del público: publicidad e información.

- Las asociaciones de amigos del museo.

- El Departamento de Relaciones Públicas e Información.

- La relación directa público- museo.

- Horarios y entradas.

- Área de acogida: información y confort.

- Las salas de exhibición a público: medios para favorecer la comunicación público-objeto.

- Control sobre el visitante.

Si bien todas son sumamente relevantes y comprenden un universo de análisis particular, para la presente investigación nos interesa profundizar las estrategias de captación del público mediante los departamentos de relaciones públicas e información ${ }^{2}$, los cuales, si bien son muy diversos, tienen algunas funciones básicas en común:

- Atender al público que accede al museo: la autora (Arias, 1990), refiere a las acciones de información y canalización de críticas y sugerencias a través de encuestas, pero también se puede incluir la atención de dudas y consultas que se realizan por otros medios, como son electrónicos y telefónicos.

- Atender a la prensa o a cualquiera de los medios de comunicación (Arias, 1990, p. 181).

- Organizar campañas publicitarias orientadas no solo a anunciar la apertura de una exposición temporal sino a difundir la imagen del propio museo: "labor que puede ser canalizada a través de la prensa, radio, cine o televisión, sin olvidar carteles propagandísticos, concursos, conferencias, videos destinados a centros docentes y ese sinfín de posibilidades que ofrece una buena planificación comercial por parte del museo: venta de diapositivas, tarjetas, posters, reproducciones de las piezas que se exponen, catálogos, guías, etc.” (Arias, 1990, p. 181). En este rubro cabe destacar lo que señala Fatás

También se les llama de prensa, comunicaciones, y según el número del personal pueden ser departamento, unidades, áreas u oficinas. 
sobre que "habitualmente, los museos no disponemos de partidas presupuestarias suficientes para emprender grandes campañas de publicidad en los medios de comunicación (Fatás, 2004, p. 145).

- Entablar contactos con otras asociaciones museísticas y hasta con distintas entidades locales o de la administración central (Arias, 1990, p. 181).

- Realizar una serie de publicaciones divulgativas de las actividades programadas así como otras de mayor entidad (Arias, 1990, p. 181).

- Además de las mencionadas por Arias (1990), se puede agregar la relación con los medios de comunicación masiva o denominados mass media. Mencionada por Fatás (2004, p. 146), que refiere a la promoción a través del "convertirse en noticia". Que refiere a aprovechar los medios para promocionar sobre las novedades del museo, mediante el envío oportuno de información principalmente vía correo electrónico, pero además refiere a la anuencia de mostrarse accesible y colaborador con estos medios, cuando deseen hacer una nota de algún tema o actividad específica. Es específico, a través del publicity, que se caracteriza por ser "la información proporcionada por una fuente externa que los medios de comunicación utilizan por tener valor informativo. Se trata de un método no controlado de difundir mensajes, ya que la fuente no paga a los medios por dicha difusión (Cutlip, Center y Broom, 2006, p. 44, citado en Mateos, 2013, p. 7).

Estas funciones, como se dijo anteriormente, son básicas, pero también podrían referir a un tipo ideal de departamento de comunicaciones, es decir, podría ser que en la vida real, existan museos que cumplan con algunas o con otras que no están contempladas. Sin embargo, sirven para circunscribir el análisis y comprender mejor las acciones que en la materia hacen los museos en estudio.

\section{Método}

La investigación tuvo tres momentos y facetas: inicialmente una búsqueda de los antecedentes de investigación y del estado de la cuestión sobre las estrategias de comunicación en museos e instituciones culturales, para lo cual se utilizó la técnica de análisis de información y bibliografía de carácter nacional e internacional. En un segundo momento, se solicitó, a los museos en estudio, información clave que refiriera a la temática, que sirviera de insumo para la elaboración de los instrumentos de recolección 
de información que se haría en la tercera fase. Una vez obtenida la información, se procedió con el análisis, a fin de identificar y comparar el estado de cada museo en el tema. La tercera fase, la recolección de datos en cada museo, pasa primero por el diseño de los instrumentos, la recogida de información y el análisis de la información, en concreto, se realizaron entrevistas a profundidad a las funcionarias encargadas de los respectivos departamentos de comunicación de los museos en estudio.

Respecto a la segunda fase, relativa al análisis de la información suministrada por los museos, se refiere el hecho de que previamente se hizo una indagación para conocer si cada museo tenía una plan estratégico o de comunicación que pudiéramos utilizar como insumo o guía para diseñar la guía de entrevista a profundidad. Al respeto, solo contamos con el plan de uno de los museos, el del Museo del Banco Central; en cuanto al del Museo de Arte Costarricense lo obtuvimos una vez finalizado el trabajo de campo y, respecto al del Museo Nacional, se encontró que no posee un plan estratégico específico que estuviera contenido en un documento escrito. Esto hizo que en la tercera parte se diseñará una guía de entrevista específica para los Museos del Banco Central (que apuntaba a tener mayor información, dado que ya teníamos una línea base) y una guía estratégica que sirviera para los otros dos museos, que tenían como característica, poseer menor información, por tanto se diseñó una de carácter más exploratorio.

Es decir, la apuesta metodológica fue utilizar una estrategia cualitativa que facilitara llegar a esos elementos que permitan comprender con mayor detalle la realidad comunicativa de los museos. Asimismo, como el estudio es de carácter exploratorio, se dificultaba la ejecución de una metodología cuantitativa, que supone una estructuración mayor de los instrumentos de recolección de información. El periodo del estudio refiere a lo elaborado por los museos en los años 2012 y 2013, tomando en cuenta que el trabajo de campo se realizó durante el 2014.

\section{Lo encontrado}

Para exponer los resultados se contemplan dos partes, una que refiere a las valoraciones sobre las estrategias de comunicación y otra sobre la comunicación externa. ${ }^{3}$ En la primera se contempla la divulgación de información, medios

\footnotetext{
Dentro de la investigación se trabajó una parte que buscaba indagar sobre las estrategias y actividades de comunicación que ejecutan los museos en los siguientes ejes: accesibilidad, grupos sociales en exclusión, equidad de género y ambiente. No obstante, esta información por razones de espacio se trabajará en otra publicación.
} 
informativos y públicos prioritarios; en la segunda, lo referido a las bases de datos, redes sociales, medios de comunicación masiva (noticieros, periódicos, radio) y la relación del museo con otros entes culturales, instituciones u organizaciones. A continuación se presenta la información por cada museo.

\section{Museos del Banco Central de Costa Rica}

Los Museos del Banco Central tienen un plan estratégico formulado desde el año 2012. Contempla tres áreas estratégicas que son: comunicación externa, comunicación interna, promoción y publicidad. La elaboración de planes estratégicos de comunicación se ha planteado, por un lado, como una necesidad para hacer acciones tomando en cuenta los perfiles y necesidades de las personas que lo visitan y que participan en sus actividades y talleres y, por el otro lado, lo formulan respondiendo a las posibilidades presupuestarias de la institución, así como acoplándose a los tiempos de planificación institucional.

Para el periodo en estudio, los museos cuentan con una encargada de comunicación, que atiende el área de relaciones públicas, asumiendo todas las actividades relativas o concernientes al área.

Respecto a las estrategias de comunicación, cabe destacar que los Museos del Banco Central han implementado una serie de acciones que se puntualizan a continuación:

- Promoción de ventas: por ejemplo con la tienda del museo.

- Publicidad.

- Refrescar la imagen gráfica del museo y posicionarla (en coordinación con el área de diseño).

- Sitio web del museo.

- Sistemas de envío masivo por medio del correo electrónico o mercado directo.

- Desplegable institucional para la persona visitante.

- Afiches mensuales.

- Redes sociales: facebook y twitter (a partir del 2010).

- Rotulación externa: por ejemplo tótem de la entrada principal y una pantalla con la información de las exhibiciones temporales y permanentes.

- Activación: que refiere a actividades en las afueras o en la plaza ubicada en la parte superior del museo, tales como: conciertos en las gradas, festivales culturales, ferias, carruseles. 
Revista Universidad en Diálogo • Vol. 6, N. 2 2, Julio-diciembre 2016, pp. 161-174

ISSN 2215-2849 • EISSN: 2215-4752

DOI: http://dx.doi.org/10.15359/udre.6-2.9

- Gestión de prensa y publicity en radio, televisión, prensa escrita.

- Enlaces con empresas e instituciones vecinas. Ejemplo el Teatro Nacional, el Gran Hotel de Costa Rica, Banco Central de Costa Rica.

Estas actividades se acompañan de otros procesos como son: la continua labor de recopilación de datos de personas que asisten al museo o a sus actividades; evaluaciones cortas de satisfacción, realización y actualización de diversas bases de datos, divididas por públicos.

Respecto a los públicos que logran captar los museos, tomando en cuenta sus estrategias de comunicación, cabe recalcar que en el caso de los Museos del Banco Central el público que más llega es el especializado, sin distinción de edad, porque es la persona que le interesan los temas que trabaja el museo y también el visitante extranjero que es captado principalmente por la oferta que ofrece el Museo de Oro.

El público que menos viene, pareciera ser el público joven, ubicado en el bloque de edades que contempla la educación secundaria, a excepción del que viene porque desde el centro educativo se organizó una gira o una visita guiada.

Sobre la consulta de los públicos que quedan descubiertos se destacan dos: las personas que están fuera de la Gran Área Metropolitana, y el bloque de las personas adultas mayores, el cual se señala que es un público con un importante potencial. Al respecto, ya se hacen iniciativas para traerlas, pero se debe reforzar en el tema del horario y la oferta de las actividades. El otro público descubierto corresponde a las comunidades de escasos recursos, en menor desventaja las ubicadas dentro del área metropolitana y zonas urbanas respecto de las que están fuera o en zonas rurales.

\section{Museo Nacional de Costa Rica}

En el Museo Nacional se encontró que no se tiene un plan estratégico formal y escrito, su trabajo en el área goza de suerte de institucionalización a partir del trabajo cotidiano y rutinario, al respecto una de las entrevistadas dijo:

Debido a la experiencia y a los diferentes proyectos que se han venido trabajando se han definido como un marco de acciones en el área de comunicación que son las que nos han dado resultados positivos y que se han mantenido en el tiempo y otras que se han venido incluyendo y que han dado resultados también positivos en el tema de comunicación, entonces, se han venido, digamos, como afianzando, 
afianzando y han llegado a ser como ese abanico de estrategias y medios que ya se han institucionalizado, también como una serie de prácticas, jverdad! tal vez como las prácticas positivas, que en el área de comunicación ya se implementan de una forma rutinaria, tanto en el área de comunicaciones directamente como lo que los otros departamentos han venido aprendiendo que se deben realizar. (entrevista Museo Nacional, 2014)

Se trabaja con la planificación anual institucional como marco y en relación con los proyectos y actividades que se van desarrollando. Si bien, parece una dinámica que ha servido, la misma puede ser negativa en tanto depende de la continuidad de las personas que se han desempeñado en esa área, dado que al cambiar el personal, no se cuenta con propuestas y planes escritos que sirvan de referencia.

La encargada de diseñar y ejecutar lo correspondiente a comunicaciones es la Unidad de Comunicaciones del Museo, que forma parte o pertenece al Departamento de Proyección Museológica, el cual cuenta con una jefatura a cargo de todo lo realizado en este. Esta unidad la conforman dos personas que, en términos generales, se encargan de dos grandes temas: prensa y relaciones públicas y comunicaciones. Si bien se detectó que en el 2009 se había conformado un plan de trabajo, la premura y la cantidad de trabajo y proyectos del museo ha hecho que no exista un plan estratégico de comunicación en términos específicos, sino que se trabaje respondiendo al plan anual.

Respecto al Museo Nacional, cabe decir que la Unidad de Comunicaciones se encarga de dar apoyo a todas las actividades que realiza el Departamento de Proyección Museológica, es decir, lo relacionado a exhibiciones, actividades educativas (festivales, charlas y talleres), mantenimiento de exhibiciones y visitas en general. A su vez, brinda apoyo a otros departamentos del Museo. Se toman en cuenta dos acciones dentro de su quehacer: reafirmar la imagen positiva de la institución para que la gente se entere de acciones o noticias que hace el museo, y buscar propiciar la visitación.

Dentro de las acciones que se han implementado durante el periodo de estudio, a continuación se puntualizan las líneas de trabajo:

- Presencia en medios de comunicación masiva: mediante la elaboración de todas las comunicaciones de prensa.

- Boletín digital.

- Coordinación de entrevistas, conferencias de prensa y boletines de prensa.

- Redes sociales: Facebook, Twitter y Youtube (a partir del año 2010). 
Revista Universidad en Diálogo • Vol. 6, N. 2 2, Julio-diciembre 2016, pp. 161-174

ISSN 2215-2849 • EISSN: 2215-4752

DOI: http://dx.doi.org/10.15359/udre.6-2.9

- Publiciy en radio, televisión y prensa escrita.

- Otros medios masivos como: prensa digital, prensa internacional, blogs.

- Vallas publicitarias.

- Atención de consultas telefónicas y personales sobre información general del museo.

- Publicidad por medio de colaboraciones o alianzas en proyectos conjuntos.

- Pizarras institucionales ubicadas en puntos estratégicos del museo.

- Relación en términos de comunicación con otras instituciones como el Ministerio de Cultura, Juventud y Deporte, con el Instituto Costarricense de Turismo y Municipalidad de San José.

- Vinculación con otros departamentos de prensa de otros museos.

Estas acciones se ven acompañadas de tareas de monitoreo y seguimiento del impacto en medios, las que se realizan mediante un archivo físico y digital que se sistematiza a mano y se apoya de colaboración de estudiantes de universidad. A su vez, se trabaja en la continua actualización de las bases de datos, así como de su correspondiente actualización. Finalmente, se encargan de la atención directa e indirecta de consultas y dudas planteadas por público interesado, que se realiza tanto por vía electrónica como por vía telefónica.

En el caso del Museo Nacional, desde la oficina de comunicaciones no se cuenta con perfil o perfiles de los públicos que más visitan el museo. Y se plantea como un perfil muy general, tal como se puede ver en las siguientes citas:

Es muy diverso, es que, qué se yo, aquí llega en un mismo día decena de estudiantes como decenas de turistas y también aqui se ve mucho muchacho joven de 17, es decir, en un mismo día, entonces es súper variado. (entrevista, Museo Nacional, 2014)

Es muy amplio, porque llegan desde niños de maternal que los traen en una visita con el jardín de niños, digámoslo así, hasta turistas adultos mayores que vienen en los cruceros, porque el museo es un museo nacional, como se llama asi "Museo Nacional", y cubre el tema arqueológico y el tema histórico entonces la gente viene como buscando esa pincelada general de que cómo es Costa Rica, entonces, el turismo es muy amplio, en turismo a veces vienen muchachos de intercambio que vienen a aprender español a Costa Rica, los traen en una visita al Museo Nacional o vienen mochileros y también extranjeros asi adultos mayores, que vienen en grupos, y 
nacionales muchos grupos así organizados, como escuelas y menos colegios; vienen más escuelas que colegios y los universitarios, pero ya vienen solos. (entrevista, Museo Nacional, 2014).

\section{Museo de Arte Costarricense}

El Museo de Arte Costarricense tiene un plan estratégico de comunicación formulado desde mediados del año 2012 y es el que se ha venido implementando hasta la actualidad (2014), se encarga de ejecutarlo la Oficina de Prensa y Relaciones Públicas que se encuentra a cargo de una sola persona. Se plantea que es muy relevante contar con un plan estratégico, lo cual se pude apreciar en la siguiente cita.

Yo siento que la primera razón es para marcarle un rumbo, un norte a lo que queremos comunicar, sobre todo desde la visión del director... (Entrevista Museo de Arte Costarricense, 2014).

En el caso del Museo de Arte Costarricense, se destacan varias estrategias de comunicación que se detallan:

- Publicity en televisión, radio y prensa escrita (esta última es la más efectiva).

- Énfasis en la labor educativa: visitas guiadas con centros educativos.

- Redes sociales: Facebook, twitter y canal de Youtube.

- Página web del Museo.

- Envío de correos e invitaciones personalizados.

- Envío de invitaciones en físico.

- Invitaciones mediante vía telefónica.

- Boletín con calendario de actividades mensuales que se distribuyen por todos los medios electrónicos anteriores.

- Rotulación externa al museo (no invasiva).

- Información a "Sección de amigos" que incluye empresas, organizaciones, agencias de viajes, entre otras.

Estas actividades se acompañan de la sistemática actualización de datos de las diversas bases de datos que maneja la oficina. Además, de la atención de eventos y actividades que realiza el museo tales como: aperturas y eventos, en toda su parte divulgativa anterior y posterior.

En el Museo de Arte Costarricense, el público meta y el público que más está llegando, según la Oficina de Presa y Comunicaciones, es el público 
Revista Universidad en Diálogo • Vol. 6, N. 2 2, Julio-diciembre 2016, pp. 161-174

ISSN 2215-2849 • EISSN: 2215-4752

DOI: http://dx.doi.org/10.15359/udre.6-2.9

joven, estudiante universitario, entre los 25 y 35 años, que tiene disposición y flexibilidad horaria, lo cual se puede evidenciar en las siguientes citas:

En el marco de este periodo que precisamente ustedes están evaluando, el público meta es el joven-adulto estamos hablando de aquel estudiante universitario, en su, que podemos decir, en la mediana etapa, digamos, que está a la mitad de la carrera o una cosa así, podemos decir que está entre sus veinte tantos casi treinta... Podemos decir que tal vez en un rango de los 25 a los 35. (entrevista Museo de Arte Costarricense, 2014)

Hace como tres años nos dimos cuenta que esa era el público que se acercaba al museo, que preguntaba sobre las actividades, que escribía en la página web, que escribía en las redes sociales...el estudiante tiene más tiempo disponible porque...Tienen sus clases algunos no trabajan siempre, entonces dedican un poco más de tiempo al ocio, pero al ocio culturalmente responsable, es así como nosotros les decimos, o artístico porque ellos están involucrados con alguna actividad artística o conocen a alguien que esté involucrado artísticamente, entonces, apoyan a los compañeros, a los amigos, a las parejas, a estos los otros, o simple y sencillamente lo disfrutan en apreciación. (entrevista Museo de Arte Costarricense, 2014).

Dentro de los públicos que no se están impactando resalta el adulto mayor o de la tercera edad y la población que vive fuera del Gran Área Metropolitana. Si bien existen visitas programadas de escuelas, colegios, universidades de zonas rurales, las provincias más lejanas como Guanacaste, Puntarenas y Limón, visitan muy poco el museo.

\section{Algunas conclusiones}

En términos generales, se puede apreciar que los tres museos en estudio han implementado estrategias de comunicación muy similares entre sí, donde se pueden destacar diversas fortalezas y elementos que se plantean como retos u obstáculos por superar, a fin de mejorar y potenciar el impacto de sus estrategias de comunicación.

Una de las fortalezas es que dos de los museos (Museos del Banco Central y Museo de Arte Costarricense) cuentan con una planificación pensada en correspondencia con las necesidades y objetivos del área de la comunicación, tomando en cuenta las necesidades de los públicos y del museo. 
En términos generales, los tres museos muestran similitudes en sus formas de operar y desarrollar sus estrategias de comunicación: las acciones como el publicity (que es más económico que la publicidad), los envíos masivos (que son más efectivos que lo impreso) y actualización de redes sociales.

Los tres museos muestran una anuencia e importante acumulación de experiencia en el manejo de medios de comunicación y redes sociales, que permite dinamizar el museo mismo y la información que se difunde y divulga, llegando a sectores o poblaciones específicas pero en ocasiones clave, por ejemplo: el público joven del Museo de Arte Costarricense.

Dentro de los retos u obstáculos que se pueden visualizar en el diseño y ejecución de estrategias de comunicación por parte de los museos, se pueden nombrar los siguientes:

- La necesidad de monitorear y evaluar lo ejecutado y realizado, a fin de verificar que efectivamente se está llegando a las poblaciones meta. Asimismo, desarrollar mecanismos sencillos y prácticos que permitan medir el impacto de las acciones emprendidas, sobretodo en redes sociales, que son las que tienen mayor dinamismo y actualización.

- Hace falta clarificar cuáles son los públicos y los perfiles de los públicos que efectivamente están llegando al museo con el fin de direccionar o canalizar los esfuerzos de comunicación hacia esos públicos o hacia otros nuevos.

- La inserción dentro de las redes sociales y medios tecnológicos es bastante reciente en los tres museos (año 2010), de manera que la evaluación y medición del impacto se hace fundamental a fin de verificar que las acciones efectuadas desde esta área están siendo efectivamente provechosas, y que no se están descuidando otros frentes comunicativos.

- El poco personal que se encarga de las labores de prensa, comunicaciones o publicidad constituye un reto, dado que se ha visualizado la cantidad de labores que se ejecutan y que son relevantes para el quehacer museístico.

- Finalmente, queda como reto plantear estrategias de comunicación que impacten a esas poblaciones ya detectadas como débiles de los tres museos, para lo cual se hace necesario el trabajo articulado con otras oficinas y departamentos de los museos, como son el de educación y el de proyección museológica. 
Revista Universidad en Diálogo • Vol. 6, N. 2 2, Julio-diciembre 2016, pp. 161-174

ISSN 2215-2849 • EISSN: 2215-4752

DOI: http://dx.doi.org/10.15359/udre.6-2.9

\section{Referencias}

Arias, L. (1990). El papel del público en el museo de hoy. Boletín de la ANABAD, Tomo 40, $\mathrm{N}^{\circ}$ 2-3, 179-188.

Bourdieu, P. y Alain, D. (2003). El amor al arte. Los museos europeos y su público. Barcelona: Paidós.

Bourdieu, P. (1984). Sociología y cultura. México: Grijalbo.

Bourdieu, P. y Loic, W. (1995). Respuestas por una antropología reflexiva. México: Grijalbo.

Bourdieu, P., Chamboredom, J.C., y Passeron, J-C. (1996) El oficio del sociólogo. México D.F.: Editorial Siglo XXI Editores,

Consejo Internacional de Museos (2007) Estatutos del ICOM. Recuperado de http://icom.museum/

la-organizacionestatutos-del-icom/3-definiciones-de-terminos/L/1/

Elias, N. (2006). Sociología fundamental. España: Editorial Gedisa.

Fatás, P. (2012). Estrategias de comunicación en museos: El caso del Museo de Altamira. VII Jornada de Museología.

Forteza, M. (2012). El papel de los museos en las redes sociales. España: Universidad de las Islas Baleares.

Hernández, R., Fernández, C. y Pilar, B. (2004) Metodología de la investigación ( $3^{\circ}$ edic.). Chile: McGrawHill Interamericana

Mateos, S. (2013). Museos y medios de comunicación. Claves, estrategias y herramientas para establecer y mantener una relación vital para los museos. Revista semestral e-rph. Recuperado de www.revistadepatrimonio.es.

Rojas, R. (1989). Guía para realizar investigaciones sociales. España: Plaza y Valdés

Rojas, R. (1989). Guía para realizar investigaciones sociales. España: Plaza y Valdés

Salazar, A., Madrigal, Á. y Delgado, A. (2011). Evaluación Interdisciplinar de museos nacionales. Un análisis del rol que cumplen los museos en la construcción de la sociedad costarricense mediante la incorporación en su quehacer comunicacional y de accesibilidad, de cuatro ejes transversales de acción esenciales para el desarrollo de nuestra sociedad: equidad de género, acceso de grupos sociales marginados, personas con capacidades diferenciadas y conservación del ambiente. Propuesta de proyecto FIDA 2012. Heredia: Universidad Nacional.

Sierra, R. (1992). Técnicas de investigación social: Teoría y ejercicios. Madrid, España: Editorial Paraninfo. 